\title{
Review of: "A megastudy of text-based nudges encouraging patients to get vaccinated at an upcoming doctor's appointment"
}

mala arora

Potential competing interests: The author(s) declared that no potential competing interests exist.

This is a US based study that evaluated the boost in flu vaccination rates by sending text reminders to patients prior to their doctors appointment. It is a single city based study covering two health care systems over a short period of 3 months. The time period of the study is too short to make valid conclusions. It should also be tested on a wider cross-section of population rather than just two health care systems.

However it is a relevant to study in todays context. Governments and health care systems wish to know which strategy will be effective in boosting the vaccination rate for Covid 19 to achieve herd immunity. The current vaccination rates in US are around $50 \%$ despite the vaccine being freely available.

1. The authors have studied various modes of text e.g Formal, casual, video based questionnaire and cartoon illustrated message. The best mode was one that was framed like an official reminder of the upcoming appointment and was personalised as in stating that " a vaccine is reserved for you at your next appointment."

2. The timing of reminders has also been studied, the best in their model being 72 hours and 24 hours before appointment. Other timings were just 72 hours before, 24 hours before, half an hour before, 10 minutes before etc. A double reminder is more reinforcing than a single one. However multiple messaging may prompt individuals to opt out of the service.

3. There was no difference in response to the text message, based on gender, age group and ethnicity although the predominant population studied was white.

Overall the boost in vaccination noted was rather small - 11\%. However it still is a useful strategy to use as it is cost effective and an efficient personal reminder.

This service can be safely expanded to other health care delivery systems for both the flu vaccine and the covid 19 vaccine. Sending a text reminder for all appointments over an extended period of at least a year should improve the results by covering a wider population. 